\title{
Diabetes: como as funções neurocognitivas influenciam na adesão ao tratamento
}

\author{
Letícia M. G. Japiassú, ${ }^{1 *}$ Simone H. Castro, ${ }^{1}$ Pricila Cristina Ribeiro, ${ }^{1}$ Gilberto N. O. Brito ${ }^{2,3}$
}

\section{Resumo}

A presente revisão tem como objetivo apresentar evidências sobre o impacto dos mecanismos fisiopatológicos do diabetes tipo1(DM1) e 2(DM2) no cérebro com repercussão importante na função neurocognitiva, e como esta repercussão interfere na adesão ao tratamento. No DM1, episódios de hiper e hipoglicemia durante o desenvolvimento provocam alterações estruturais e metabólicas no cérebro, enquanto que no DM2 um estado de relativa resistência insulínica associado à diminuição da atividade da enzima de degradação de insulina no cérebro levaria à degeneração neuronal. Tanto no DM1 quanto no DM2, a agressão ao neurônio provocada pelos mecanismos fisiopatológicos do diabetes provoca fraturas no funcionamento neurocognitivo, que podem se apresentar como dificuldades da atenção, dismnesia e síndrome disexecutiva. Como o tratamento do diabetes é bastante complexo, estas dificuldades neurocognitivas interagindo com as dificuldades intrínsecas (pré-mórbidas) prejudicam a adesão do paciente ao tratamento. Como enfatizado neste artigo, o analfabetismo, pleno e funcional, representa um dos mais importantes fatores pré-mórbidos que dificulta esta adesão. A estratégia de tratamento de pacientes diabéticos, independentemente do tipo, inclui um esquema complexo de orientações medicamentosas e não medicamentosas que devem ser implantadas no seu cotidiano. Educação em saúde é fundamental para o eficaz manejo de qualquer doença e, mais ainda, do diabetes. De forma mais ampla, indivíduos com pouca escolaridade mostram baixo entendimento sobre a própria doença e, consequentemente, pior autocuidado e condição clínica. Cabe ao provedor principal de saúde determinar a forma mais adequada de apresentar a estratégia de tratamento através do conhecimento do perfil pré e pós-mórbido das funções neurocognitivas e também das características intrínsecas (ex.: analfabetismo, personalidade) ao paciente. O dilema atualmente imposto ao sistema de saúde é como fazer chegar ao paciente diabético os benefícios dos avanços científicos na área, apesar de toda a complexidade da estratégia do tratamento.

Descritores: Diabetes mellitus; Fisiopatologia; Atuação (Psicologia); Terapia comportamental; Mortalidade.

\section{Abstract \\ Diabetes: how neurocognitive functions influen- ce treatment adherence}

In this paper, we review the evidence on the repercussion of the pathophysiology of diabetes type 1 and 2 in the brain and its impact on neurocognitive function and thus on adherence to treatment. Episodes of hyper and hypoglycemia during de-
1. Disciplina de Diabetes. Departamento de Medicina Interna. Universidade do Estado do Rio de Janeiro. Rio de Janeiro, RJ, Brasil.

2. Departamento de Psiquiatria e Saúde Mental. Instituto de Saúde Coletiva. Universidade Federal Fluminense. Niterói, RJ, Brasil.

3. Departamento de Pesquisa. Instituto Nacional de Saúde da Mulher, da Criança e do Adolescente Fernandes Figueira. Fundação Oswaldo Cruz. Rio de Janeiro, RJ, Brasil.

*Endereço para correspondência:

Serviço de Diabetes e Metabologia, HUPE, UERJ

Boulevard 28 de Setembro, 77, 3ㅇandar

Rio de Janeiro, RJ, Brasil. CEP: 20551-030.

E-mail: leticiamauricio@gmail.com

Revista HUPE, Rio de Janeiro, 2015;14(4):37-42

doi: $10.12957 /$ rhupe.2015.20042

Recebido em 28/11/2014. Aprovado em 13/01/2015.

velopment in DM1 cause structural and metabolic changes in the brain whereas in DM2 a state of relative insulin resistance and decreased activity of the insulin degrading enzyme in the brain would lead to neuronal degeneration. Both, in DM1 and DM2, neuronal insults due to the pathophysiological mechanisms of diabetes induce neurocognitive behavioral fractures that may present as difficulties in attention, dismnesia and disexecutive syndrome. Since the treatment of diabetes is highly complex, disease-related neurocognitive difficulties in interaction with intrinsic patient-related (premorbid) characteristics obliterate compliance to treatment. Illiteracy is one of the most important patient-related (pre-morbid) characteristics that frequently makes treatment adherence fragile. The treatment of diabetes, regardless of type, involves a complex daily routine of medical and non-medical instructions. Health education is fundamental to the effective management of any disease and, even more, of diabetes. Patients with fewer years of education show limited understanding of the disease and, as a consequence, worse self-care and clinical condition in comparison with patients with more years of education. The health provider must determine the most appropriate way to present the treatment strategy through knowledge of patient's neurocognitive functions and his (or her) intrinsic (premorbid) characteristics such as literacy and personality. The dilemma now presented to the health care system is how to deliver to the diabetic patient the benefits of scientific advances in the field despite the complexity of the treatment strategy.

Keywords: Diabetes mellitus; Physiopathology; Acting out; Behavior therapy; Mortality. 


\section{Resumen}

Diabetes: cómo las funciones neurocognitivas influyen en la adhesión al tratamiento

Esta revisión tiene como objetivo proporcionar evidencias sobre el impacto de los mecanismos fisiopatológicos de la diabetes tipo 1(DM1) y 2 (DM2) en el cerebro, con repercusión importante en la función neurocognitiva, y cómo esta repercusión interfiere en la adhesión al tratamiento. En DM1, los episodios de hiper e hipoglucemia durante el desarrollo causan alteraciones estructurales y metabólicas en el cerebro, mientras que en DM2, un estado de relativa resistencia a la insulina asociado con la disminución de la actividad de la enzima de degradación de insulina en el cerebro, conduciría a la degeneración neuronal. Tanto en DM1 como en DM2, la agresión a las neuronas provocada por los mecanismos fisiopatológicos de la diabetes causa fracturas en el funcionamiento neurocognitivo, que pueden presentarse como dificultades de atención, dismnesia y síndrome disejecutivo. Como el tratamiento de la diabetes es muy complejo, estas dificultades neurocognitivas interactuando con las dificultades intrínsecas (premórbidas) causan perjuicio en la adhesión del paciente al tratamiento.

\section{Introdução}

A acentuada morbidade associada ao diabetes mellitus, seja do tipo 1 (DM1) seja do tipo 2 (DM2), repercute na qualidade de vida do paciente pelas mais variadas complicações decorrentes dos seus processos fisiopatológicos, especialmente quando se tornam crônicos. Avanços no conhecimento dos mecanismos envolvidos na fisiopatologia do DM1 e DM2 abrem novas perspectivas no tratamento medicamentoso e também progressivamente aumentam o nível de complexidade para o correto seguimento das recomendações terapêuticas. O dilema atualmente imposto ao sistema de saúde é como fazer chegar ao paciente diabético os benefícios dos avanços científicos na área, apesar de toda a complexidade da estratégia do tratamento.

Embora a evidência indique que uma adequada adesão à estratégia de tratamento se associe a um melhor controle das complicações decorrentes dos processos fisiopatológicos associados ao diabetes, ${ }^{1,2}$ a complexidade das recomendações (conforme mostra a Tabela 1) torna muito difícil a adesão.

\section{Análise neurocognitiva das recomendações de tratamento do diabetes}

Para dar início ao tópico em epígrafe, é necessário
Como se destaca en este artículo, el analfabetismo absoluto y funcional, es uno de los factores pre-mórbidos más importantes que dificulta esta adhesión. La estrategia de tratamiento de los pacientes diabéticos, independientemente del tipo, incluye un esquema complejo de orientaciones farmacológicas y no farmacológicas que deben ser introducidas en su vida diaria. La educación en salud es fundamental para el manejo eficaz de cualquier enfermedad y, más aún, de la diabetes. En términos más generales, los individuos con baja escolaridad demuestran poca comprensión sobre su propia enfermedad y, en consecuencia, peor autocuidado y condición clínica. Corresponde al proveedor principal de salud determinar la forma más adecuada de presentar la estrategia de tratamiento a través del conocimiento del perfil pre y post-mórbido de las funciones neurocognitivas y también según las características intrínsecas (por ejemplo: analfabetismo, personalidad) del paciente. El dilema actualmente impuesto al sistema de salud es cómo hacer llegar al paciente diabético, los beneficios de los avances científicos en el área, a pesar de toda la complejidad de la estrategia del tratamiento.

Palabras clave: Diabetes mellitus; Fisiopatología; Rendimiento (Psicología); Terapia conductista; Mortalidad.

considerar alguns pressupostos. Primeiro, o exame reflete as funções do paciente em um determinado corte transversal no tempo e pouco significa se não incorporar a dinâmica dos resultados obtidos. Segundo, além da história ontogenética do paciente, o profissional da saúde não pode perder a perspectiva de que aquele organismo também tem uma história filogenética, conforme já abordado por um dos autores do presente artigo. $^{3}$

Isto posto, observando-se a lista de recomendações propostas para o tratamento do diabetes, conforme ilustrado na tabela 1, e imaginando-se o encontro do paciente com o seu médico, verifica-se que a análise neurocognitiva tem que começar, necessariamente, pelo bom funcionamento dos sistemas sensoriais, em especial o auditivo e o visual. Comprovada a preservação de função auditiva e visual, torna-se importante determinar a capacidade de processamento de informação apresentada por via oral e também escrita, porque é através destes dois canais que o profissional de saúde procura transmitir a essência das recomendações que devem ser seguidas pelo paciente. Em outras palavras, é necessário que se determine o nível de alfabetização do paciente.

Em seguida, é importante examinar a vigilância, atenção, orientação temporoespacial, memória (verbal, visual, imediata e tardia), coordenação motora fina, práxis, flexibilidade cognitiva e também estimar 
a função intelectiva do paciente. Pesquisa em andamento conduzida pelo Grupo de Neurodiabetologia do nosso Serviço contempla a avaliação de todas estas funções neurocognitivas através de um instrumento em desenvolvimento, o Exame Neurocognitivo do Rio de Janeiro (ENCORJ). Ademais, há que se examinar o impacto psicossocial do diagnóstico de diabetes através de instrumentos de rastreio de depressão e ansiedade, também incluídos no nosso protocolo de exame.

É preciso enfatizar que o impacto neurobiológico da fisiopatologia do diabetes interage ao longo do tempo com uma arquitetura neural já presente no paciente antes do início da doença.

\section{Impacto neurobiológico da fisiopatologia do diabetes e repercussão na função neurocognitiva}

O paciente diabético no momento do diagnóstico provavelmente já sofreu o impacto de vários anos de alterações metabólicas e agressões decorrentes dos processos fisiopatológicos da doença, que incluem ataques indiretos (e.g., vasculares) e diretos (e.g., hipoglicemia, glicotoxicidade, modulação de receptores) ao neurônio. Por óbvio, estes ataques ao neurônio provocam alterações na circuitaria neural e, consequentemente, no funcionamento do sistema nervoso, incluindo fraturas na função neurocognitiva.

Bade-White e Obrzut ${ }^{4}$ apresentaram revisão sobre o impacto neurocognitivo do DM1 em crianças e adultos jovens, e relataram alterações importantes na memória, atenção, habilidades motoras, capacidade visoespacial, funcionamento executivo e desempenho acadêmico em particular nos pacientes com início precoce da doença (antes dos cinco anos de idade) e com história de episódios recorrentes de hipoglicemia. Ademais, estes autores também relataram que pacientes DM1, novamente em especial os de início precoce, apresentam alterações estruturais no cérebro compatíveis com atrofia como, por exemplo, ventriculomegalia, além de microangiopatias tipicamente observadas na substância branca, que se correlacionam com deficit neurocognitivos. Profissionais de saúde devem estar sempre atentos à possibilidade de que problemas de atenção se relacionem a episódios de hipoglicemia decorrentes do DM1 e não a outras nosologias mais reconhecidas na infância, como o transtorno de hiperatividade e deficit de atenção.

Como a fonte de energia principal do cérebro é a glicose, não surpreende que a diminuição dos seus níveis no sangue cause prejuízos nas funções cognitivas. ${ }^{5}$ Alguns estudos mostram que o córtex cerebral e o hipocampo, estruturas intimamente envolvidas em processos cognitivos, são mais sensíveis a episódios de hipoglicemia e, inversamente, que o tronco cerebral e a medula, estruturas mais relacionadas à função autonômica, são mais resistentes. ${ }^{4}$ Episódios de hipoglicemia durante o desenvolvimento podem ter efeitos ainda mais deletérios no cérebro ${ }^{6}$ e, por isso, a idade do início da doença parece ter importante papel no funcionamento neurocognitivo do paciente DM1.

A associação do DM2 com a doença de Alzheimer (DA) vem se tornando reconhecida, e a sua importância, por se tratar de duas das mais prevalentes doenças crônicas que acometem idosos, é indiscutível do

Tabela 1. Resumo das recomendações de tratamento do paciente diabético.

\begin{tabular}{l|l}
\hline \multicolumn{1}{c}{ Medidas não farmacológicas } & \multicolumn{1}{c}{ Medidas farmacológicas } \\
\hline $\begin{array}{l}\text { Prática regular de atividade física } \\
\text { Dieta (quantidade a ser ingerida, qualidade do alimento, } \\
\text { horários das refeições) }\end{array}$ & Uso de hipoglicemiantes orais (antes ou após refeições) \\
\begin{tabular}{l|l} 
Automonitorização de glicemia capilar & Insulina rápida (30 minutos ou menos antes das refeições, de \\
Cuidado com os pés & acordo com o tipo de insulina)
\end{tabular} \\
$\begin{array}{l}\text { Consultas multiprofissionais (nutricionista, endocrinologista, } \\
\text { oftalmologista, cardiologista, entre outros, caso se aplique) }\end{array}$ & $\begin{array}{l}\text { Ajuste de dose de insulina, de acordo com glicemia capilar ou } \\
\text { contagem de carboidrato }\end{array}$ \\
\hline
\end{tabular}




\section{Artigo de revisão}

ponto de vista epidemiológico. ${ }^{3}$ Estudos e pesquisas mostram que existe no cérebro do paciente com DA um estado de relativa resistência à insulina que levou ao conceito de diabetes do tipo 3 (DM3), preconizado por alguns investigadores, como de la Monte e colaboradores, ${ }^{7}$ na tentativa de esclarecer os fundamentos neurobiológicos desta associação. Em excelente e recente revisão sobre o tema, Moreira e colaboradores $^{8}$ discutiram a relevância de vários outros fatores, como a diminuição na captação neuronal de glicose, a elevação na produção e secreção de amiloide com formação de placas neuríticas e hiperfosforilação da proteína tau secundários à resistência insulínica, além de diminuição da atividade da enzima de degradação da insulina e aumento do estresse oxidativo secundário à hiperglicemia. O conjunto destes vários elementos de neuroagressão no decorrer dos anos levaria a deficit no funcionamento neurocognitivo e, eventualmente, a quadros demenciais do tipo Alzheimer, tornando ainda mais difícil a adesão ao tratamento.

A comparação dos achados neurobiológicos no DM1 e DM2 revela similaridades no impacto neuromorfofuncional das duas doenças apesar das conhecidas diferenças nas respectivas fisiopatologias, conforme discutido acima. Por exemplo, tanto no DM1 quanto no DM2 em determinado momento da evolução da doença há evidência de neurodegeneração com aumento do espaço ventricular cerebral que se acompanha de alterações neurofuncionais em domínios cognitivos, especialmente na atenção, memória e funções executivas. Neste caso, poderia se supor que tanto no DM1 quanto no DM2 a vulnerabilidade a quadros demenciais fosse equivalente, mas seria mais evidente no DM2 e não no DM1 pela maior mortalidade em idades mais precoces no DM1. Espera-se que novos estudos iluminem esta importante questão.

\section{Adesão ao tratamento e sua importância no manejo da doença}

Adesão, como definido na literatura, é quando o comportamento do paciente (uso de medicações, dieta e mudança de estilo de vida) coincide com a orientação médica ou de outro profissional da área de saúde. ${ }^{9} \mathrm{~A}$ baixa adesão ao tratamento tem sido mencionada na literatura como um importante problema enfrentado pelos profissionais de saúde, ${ }^{10}$ e é frequentemente observada em situações que requerem tratamentos longos, complexos e quando alterações no estilo de vida do paciente precisam ser realizadas nos casos de doenças crônicas, como o diabetes.

Estudos como o Diabetes Control and Compli- cations Trial (DCCT) e United Kingdom Prospective Study (UKPDS) $)^{1,2}$ mostram a importância da adesão ao tratamento na prevenção de complicações crônicas relacionadas ao diabetes. Entretanto, outras pesquisas indicam que pacientes diabéticos de maneira geral têm baixa adesão ao tratamento ${ }^{11}$ e que apenas cerca de $7 \%$ dos pacientes diabéticos seguiram integralmente $\mathrm{o}$ tratamento proposto. ${ }^{12}$ Além disso, outro relato revelou uma taxa de adesão de $20 \%$ à correta e regular aplicação de insulina. ${ }^{13}$ Espera-se que uma prescrição mais complexa provavelmente provoque uma menor adesão ao tratamento e que os pacientes com melhor adesão ao tratamento tenham um melhor controle metabólico. ${ }^{14}$

De acordo com alguns pesquisadores, ${ }^{4}$ a adesão ao tratamento requer que o paciente: (i) possua em seu repertório os comportamentos necessários à adesão; (ii) tenha informações sobre as razões que tornam necessários esses comportamentos; (iii) discrimine em que situações determinados comportamentos devem ser emitidos; (iv) disponha de um repertório comportamental alternativo; (v) tenha em seu repertório comportamentos de busca por informações.

Dentre os diversos fatores relativos ao paciente, merece nota o conceito de loco de controle, que reflete o que ele (ou ela) considera como o nível de controle que exerce sobre a sua própria vida, conforme exposto originalmente por Rotter ${ }^{15}$ e estendido para questões de saúde por Rodin. ${ }^{16}$ De acordo com este conceito, pacientes com loco de controle predominantemente externo, i.e., com a tendência a atribuírem a outrem (como, por exemplo, o profissional de saúde) o controle das suas vidas apresentam pior adesão a tratamento em comparação com pacientes com loco de controle predominantemente interno, i.e., com a tendência a eles próprios exercerem o controle sobre suas vidas. No que concerne ao tratamento do diabetes, há evidência consistente com a importância do loco de controle, ${ }^{17,18}$ mas também contrária. ${ }^{19}$

Outra abordagem com enfoque na relação entre o paciente e o profissional de saúde tem como fulcro conceitual a teoria do desenvolvimento do vínculo com o provedor principal na infância e mostra que pacientes com história de vínculo satisfatório apresentam uma melhor adesão ao tratamento do diabetes e melhor controle metabólico em comparação com aqueles sem história de vínculo satisfatório. ${ }^{20}$ Este trabalho indica claramente a possibilidade de que fatores relativos ao desenvolvimento ontogenético do paciente influenciem na vida adulta a sua adesão ao tratamento do diabetes. 


\section{Alfabetização e adesão ao tratamento}

A Organização Mundial de Saúde (OMS/WHO World Health Organization) em 1998 definiu "literacia em saúde" como o conjunto de habilidades cognitivas para determinar a motivação e a aptidão dos indivíduos para terem acesso, entenderem e usarem informações de forma a promover e manter boa saúde. ${ }^{21}$

Pacientes com dificuldades na compreensão da leitura (literacia) e de informação numérica (numeracia) frequentemente apresentam dificuldades na compreensão de receitas médicas ou bulas de medicações, interpretação de resultados de exames e testes de glicemia capilar, entendimento de esquemas de aplicação de insulina, principalmente o de bolus pré-prandial com insulinas de ação rápida, além de dificuldade para compreender recomendações médicas, folhetos educativos ou termos de consentimento. Trabalho recente desenvolvido no nosso Serviço e apresentado no Congresso da Sociedade Brasileira de Endocrinologia e Metabologia (SBEM) em 2013 revelou que mais de um terço dos nossos pacientes apresentavam analfabetismo pleno ou funcional. Seria factível esperar que esses pacientes mostrem um entendimento mais limitado sobre a sua doença e, por conseguinte, tenham também maior dificuldade no gerenciamento das várias contingências relacionadas ao tratamento e apresentem maior probabilidade de hospitalização decorrente de complicações. ${ }^{22,23}$ Embora a hipótese de associação entre baixa alfabetização e pior controle clínico no diabetes tenha valor heurístico, os dados ainda são controversos. Por exemplo, Williams e colaboradores mostraram que, apesar de robusta associação entre alfabetização e entendimento da doença, não foi encontrada uma relação significativa entre alfabetização e controle clínico. ${ }^{23}$

Por outro lado, há dados que sugerem que pacientes analfabetos plenos não mostram pior controle clíni$\mathrm{co}$, talvez porque consigam desenvolver estratégias compensatórias das suas dificuldades, especialmente se inicialmente também reconhecerem as suas limitações. ${ }^{24}$ Pacientes com baixa escolaridade procuram esconder as suas dificuldades na leitura dos seus cônjuges, familiares e amigos, podendo desenvolver estratégias compensatórias. $^{25}$

\section{Perspectivas para uma maior adesão do paciente diabético ao esquema de tratamento}

Como as evidências discutidas anteriormente neste artigo sugerem, qualquer abordagem com o objetivo de alavancar a adesão de pacientes diabéticos às diversas recomendações medicamentosas e não medicamentosas do seu tratamento precisa contemplar a relevância de dois grandes tipos de variáveis: (i) intrínsecas ou constitucionais do paciente, que independem a priori do impacto neuromorfofuncional dos processos fisiopatológicos da doença (e.g., loco de controle, vínculo com o provedor principal de saúde, alfabetização/escolaridade); e (ii) decorrentes ou secundárias ao impacto neurobiológico dos processos fisiopatológicos da doença em interação com variáveis intrínsecas (e.g., atenção, memória, flexibilidade cognitiva). Embora muitas vezes investigadores não se refiram com clareza a esses dois tipos de variáveis, elas estão sempre inseridas em qualquer estudo ou pesquisa sobre adesão a tratamento.

A adesão ao tratamento faz parte de um movimento (comportamento) do paciente em relação a si mesmo, aos seus conceitos de vida e doença, ao entendimento do diabetes em sua plena complexidade, incluindo o tratamento. Há a necessidade de se desenvolver um aparato mental, no qual se inclui a alfabetização e escolaridade, para lidar com uma nova linguagem (jargão médico, novo vocabulário, nova rotina, cálculos matemáticos, lógica, abstração, memória, dentre outros). Entende-se que os pacientes, nas tomadas de decisão, variam entre a internalidade absoluta à externalidade total. ${ }^{26}$ Aqui fica clara a importância do desenvolvimento do loco de controle e também do vínculo com o provedor principal (de saúde). Além dos aspectos relacionados a variáveis intrínsecas aqui discutidas, devemos também incorporar variáveis relacionadas ao impacto neurocognitivo do insulto neural associado à fisiopatologia do diabetes.

Pode-se oferecer ao paciente caminhos diversos, ferramentas variadas no cuidado com a doença, cujo foco seria fazer uso de instrumentos que são externos ao seu controle; e também meios para aprender o diabetes a partir do autorreconhecimento como diabético, cujo foco (interno), por sua vez, seria tomar decisões sobre si mesmo. Estaríamos, desta forma, ao mesmo tempo estimulando o pensar a doença a partir de fatores internos, em que a crença do paciente é a de que ele próprio controla o seu estado de saúde, como também o pensar que fatores externos, incluindo os relativos a vínculo, têm influência no controle da doença (médicos, enfermeiros, amigos, familiares, clínica, posto de saúde) e, mais amplamente, políticas públicas de largo alcance que estimulem mudanças de hábitos (e.g., atividade física).

Tradicionalmente, o material educacional é denso de informações, prejudicando a compreensão de pon- 
tos importantes por parte daqueles indivíduos com dificuldades, mesmo que sutis, na alfabetização, muitas vezes sem que o profissional de saúde suspeite da baixa capacidade de compreensão da leitura do seu paciente. Outro importante fator na adesão ao tratamento é o nível de entendimento da própria doença (conhecimento sobre diabetes) e também do tratamento. Infelizmente, há dados que mostram que apenas o conhecimento das instruções fornecidas pelos profissionais de saúde não garante a adesão do paciente. ${ }^{27}$

Para assegurarmos uma melhor adesão do paciente diabético, são necessárias medidas educacionais que informem o paciente sobre a sua doença e possíveis complicações, esclarecer dúvidas, mostrar a forma correta de tomar a medicação (antes ou após uma refeição, por exemplo) e também o uso correto de seringas ou canetas de insulina. Medidas educativas também facilitam a aceitação da doença por parte do paciente e o entendimento das modificações comportamentais necessárias para o tratamento. ${ }^{28}$ Por fim, deve-se refletir sobre a implantação de políticas públicas nos moldes descritos por Bonita e colaboradores ${ }^{29}$ na promoção e manutenção da saúde.

\section{Agradecimentos}

Os autores agradecem aos pacientes e a toda equipe doServiço de Diabetes da UERJ pela colaboração com os nossos estudos e pesquisas e ao apoio da FAPERJ através de uma bolsa de treinamento e capacitação técnica (E26/103.452/2012) à segunda autora.

\section{Referências}

1. Turner RC, Millns H, Neil HA, et al. Risk factors for coronary artery disease in non-insulin dependent diabetes mellitus: United Kingdom Prospective Diabetes Study (UKPDS: 23). BMJ. 1998 Mar 14;316(7134):823-8.

2. AADE Guidelines for the Practice of Diabetes Self-Management Education and Training (DSME/T). The Diabetes Educator. 2009;35.

3. Brito GN. Exercise and cognitive function: a hypothesis for the association of type II diabetes mellitus and Alzheimer's disease from an evolutionary perspective. Diabetol Metab Syndr. 2009 Sep 18;1(1):7. doi: 10.1186/1758-5996-1-7.

4. Bade-White PA, Obrzut JE. The neurocognitive effects of type 1 diabetes mellitus in children and young adults with and without hypoglycemia. J Dev Phys Disabil. 2009;21:425-40. doi:10.1007/s10882-009-915

5. Sommerfield AJ, Deary IJ, McAulay V, et al. Moderate hypoglycemia impairs multiple memory functions in healthy adults. Neuropsychology. 2003 Jan;17(1):125-32.

6. McCall AL. The impact of diabetes on the CNS. Diabetes. 1992 May;41(5):557-70.

7. de la Monte SM, Tong M, Lester-Coll N, et al. Therapeutic rescue of neurodegeneration in experimental type 3 diabetes: relevance to Alzheimer's disease. J Alzheimers Dis. 2006
Sep;10(1):89-109.

8. Moreira RO, Campos SC, Soldera AL. Type 2 Diabetes Mellitus and Alzheimer's Disease: from physiopathology to treatment implications. Diabetes Metab Res Rev. 2013 Jul 18. doi: 10.1002/dmrr.2442. [Epub ahead of print]

9. Haynes RB, Sackett DL, Taylor DW. Compliance in health care. Baltimore: Johns Hopkins University press; 1979. p.1-10.

10. Organização Mundial da Saúde. Cuidados inovadores para condições crônicas: componentes estruturais de ação: relatório mundial. Brasília: Organização Mundial da Saúde; 2003.

11. Kurtz SM. Adherence to diabetes regimens: empirical status and clinical applications. Diabetes Educ. 1990 JanFeb;16(1):50-9.

12. Cerkoney KA, Hart LK. The relationship between the health belief model and compliance of persons with diabetes mellitus. Diabetes Care. 1980 Sep-Oct;3(5):594-8.

13. Watkins JD, Roberts DE, Williams TF, et al. Observation of Medication Errors Made by Diabetic Patients in the Home. Diabetes. 1967; 16:882-5.

14. McNabb WL. Adherence in diabetes: can we define it and can we measure it? Diabetes Care. 1997 Feb;20(2):215-8.

15. Rotter JB. Generalized expectancies for internal versus external control of reinforcement. Psychological Monographs: General and Applied. 1966;80:1-28.

16. Rodin J. Aging and health: effects of the sense of control. Science. 1986 Sep 19;233(4770):1271-6.

17. Macrodimitris SD, Endler NS. Coping, control, and adjustment in Type 2 diabetes. Health Psychol. 2001 May;20(3):208-16.

18. Morowatisharifabad MA, Mahmoodabad SS, Baghianimoghadam $\mathrm{MH}$, et al. Relationships between locus of control and adherence to diabetes regimen in a sample of Iranians. Int $\mathrm{J}$ Diabetes Dev Ctries. 2010 Jan;30(1):27-32.

19. Bunting BP, Coates V. Stability and predictors of blood glucose levels: an intra- and inter-individual analysis. Psychol Health Med. 2000;5:251-58.

20. Ciechanowski PS, Katon WJ, Russo JE, et al. The patient-provider relationship: attachment theory and adherence to treatment in diabetes. Am J Psychiatry. 2001 Jan;158(1):29-35.

21. World Health Organization. Health Promotion glossary. 1998 [Access on: 28 mar 2016]. Available at: http://www.who.int/ healthpromotion/about/HPR\%20Glossary\%201998.pdf

22. Quirk PA. Screening for literacy and readability: implications for the advanced practice nurse. Clin Nurse Spec. 2000 Jan;14(1):26-32.

23. Souza JG, Apolinario D, Magaldi RM, et al. Functional health literacy and glycaemic control in older adults with type 2 diabetes: a cross-sectional study. BMJ Open. 2014;4(2):e004180.

24. Diabetes Control and Complications Trial (DCCT): results of feasibility study. The DCCT Research Group. Diabetes Care. 1987 Jan-Feb;10(1):1-19.

25. Tang YH, Pang SM, Chan MF, et al. Health literacy, complication awareness, and diabetic control in patients with type 2 diabetes mellitus. J Adv Nurs. 2008 Apr;62(1):74-83.

26. Coleta JAD. A escala de lótus de controle interno-externo de Rotter: um estudo exploratório. Arq Bras Psicol. 1979;31(4):167-81.

27. Miyazaki MCOS, Domingos NAM, Caballo VE. Psicoterapias Cognitivo-comportamentais: um diálogo com a psiquiatria. Porto Alegre: Artmed; 2001.

28. Grueninger UJ. Arterial hypertension: lessons from patient education. Patient Educ Couns. 1995 Sep;26(1-3):37-55.

29. Bonita R, Magnusson R, Bovet $P$, et al. Country actions to meet UN commitments on non-communicable diseases: a stepwise approach. Lancet. 2013 Feb 16;381(9866):575-84. 